\title{
NBS Determination of the Fine-Structure Constant, and of the Quantized Hall Resistance and Josephson Frequency-to-Voltage Quotient in SI Units
}

\author{
M. E. CAGE, RONALD F. DZIUBA, MeMBer, IEEe, RANDOLPH E. ELMQUIST, \\ BRUCE F. FIELD, MEMBER, IEEE, GEORGE R. JONES, JR., \\ P. THOMAS OLSEN, MEMBER, IEEE, WILLIAM D. PHILLIPS, \\ J. Q. SHIELDS, R. L. STEINER, BARRY N. TAYLOR, FELLOW, IEEE, \\ AND EDWIN R. WILLIAMS, MEMBER, IEEE
}

\begin{abstract}
Results from NBS experiments to realize the ohm and the watt, to determine the proton gyromagnetic ratio by the low field method, to determine the time dependence of the NBS representation of the ohm using the quantum Hall effect, and to maintain the NBS representation of the volt using the Josephson effect, are appropriately combined to obtain an accurate value of the fine-structure constant and of the quantized Hall resistance in SI units, and values in SI units of the Josephson frequency-to-voltage quotient, Planck constant, and elementary charge.
\end{abstract}

\section{INTRODUCTION}

$\mathrm{T}$ COMPARE critically predictions of quantum electrodynamics (QED) with experimental results often requires an accurate value of the fine-structure constant $\alpha$ which is independent of QED. For example, a highly accurate, QED-independent value of $\alpha$ is necessary for obtaining a theoretical value of the electron magnetic moment anomaly $a_{\mathrm{e}}$ from QED for comparison with the experimentally determined value of $a_{\mathrm{e}}$ [1]

Under the assumption that the quantized Hall resistance $R_{\mathrm{H}}$ as measured using a quantum Hall effect (QHE) device is equal to $h / e^{2}$ ( $h$ is the Planck constant and $e$ is the elementary charge), there are at present two virtually independent ways of obtaining a reliable, QED-independent value of $\alpha$ from high precision experiments in the field of electrical metrology [2]. The first is to use the QHE and a direct realization of the ohm by means of a calculable capacitor to determine $R_{\mathrm{H}}=h / e^{2}$ in SI units, that is, in ohms:

$$
\alpha=\mu_{0} c / 2 R_{\mathrm{H}}
$$

where $\mu_{0}=4 \pi \times 10^{-7} \mathrm{~N} / \mathrm{A}^{2}$ exactly, is the permeability of vacuum and $c=299972458 \mathrm{~m} / \mathrm{s}$ exactly, is the speed of light in vacuum.

Manuscript received June 10,1988

The authors are with the National Bureau of Standards, Electricity Division, Gaithersburg, MD 20899.

IEEE Log number 8824970 .
The second involves the use of the relation

$$
\alpha^{3}=2 \mu_{0} R_{\infty} \gamma_{\mathrm{p}}^{\prime} /\left(\mu_{\mathrm{p}}^{\prime} / \mu_{\mathrm{B}}\right) R_{\mathrm{H}} E_{\mathrm{J}}
$$

where $R_{\infty}$ is the infinite mass Rydberg constant, $\gamma_{\mathrm{p}}^{\prime}$ is the proton gyromagnetic ratio (the prime indicates a spherical, pure $\mathrm{H}_{2} \mathrm{O}$ proton nuclear magnetic resonance or NMR sample at $\left.25^{\circ} \mathrm{C}\right), \mu_{\mathrm{p}}^{\prime} / \mu_{\mathrm{B}}$ is the magnetic moment of the proton in units of the Bohr magneton, $R_{\mathrm{H}}=h / e^{2}$ is again the quantized Hall resistance, and $E_{\mathrm{J}}$ is the Josephson frequency-to-voltage quotient and is assumed equal to $2 e / h$.

Although (1) and (2) both involve $R_{\mathrm{H}}$, they do so in very different ways. Equation (1) requires $R_{\mathrm{H}}$ in SI units, whereas (2) has the important property that it is independent of the electrical units used, that is, it holds if $\gamma_{\mathrm{p}}^{\prime}, R_{\mathrm{H}}$, and $E_{\mathrm{J}}$ are measured in SI units, or if $\gamma_{\mathrm{p}}^{\prime}$ is determined by the weak or low-field method [3] and it, $R_{\mathrm{H}}$, and $E_{\mathrm{J}}$ are measured in terms of the EMF of an arbitrary battery and the resistance of an arbitrary resistor. Because of this property, method two does not require the direct realization of an SI electrical unit. Since the two methods involve radically different experiments but can yield $\alpha$ values of comparable uncertainty, each can serve as a critical check on the other.

Equation (1) is also noteworthy. Since $\mu_{0}$ and $c$ are exact quantities, it implies that if $\alpha$ is known with a certain fractional uncertainty, $R_{\mathrm{H}}$ will be known in SI units (i.e., ohms) with the same fractional uncertainty. This is significant because the Comité Consultatif d'Électricité (CCE) of the Comité International des Poids et Mesures (CIPM) has decided to recommend that starting on January 1,1990 , practical or laboratory representations of the ohm be based on the QHE using an adopted value of $R_{\mathrm{H}}$ in agreement with the SI value. This value is to be selected by the CCE at its September 1988 meeting and is to be used by all those laboratories which choose to base their ohm representation on the QHE [4].

Similarly, the CCE has decided to recommend that starting on January 1, 1990, all those laboratories that base 
their practical or laboratory representation of the volt on the Josephson effect use a new value of the Josephson frequency-to-voltage quotient $E_{\mathrm{J}}=2 e / h$ in agreement with the SI value. This value, which is to replace the one suggested by the CCE in 1972 and used by most countries, is also to be selected by the CCE at its September 1988 meeting [4].

\section{NBS MEAsurements}

We have been carrying out a number of experiments at NBS that are now yielding results and bear on the issue of a reliable value of $\alpha$, and of $R_{\mathrm{H}}$ and $E_{\mathrm{J}}$ in SI units. These are:

1) maintenance of the NBS representation of the volt $V_{\mathrm{NBS}}$ using Josephson 1-V arrays [5];

2) determination of the NBS representation of the ohm $\underline{\Omega}_{\text {NBS }}$ (which is based on the mean resistance of five Thomas-type wire-wound resistors) in ohms using the NBS calculable capacitor [6];

3) determination of the time dependence of the NBS representation of the ohm $\underline{\Omega}_{\mathrm{NBS}}$ through comparisons with the quantized Hall resistance $R_{\mathrm{H}}$ [7];

4) determination of the NBS electrical representation of the watt $W_{\mathrm{NBS}}=V_{\mathrm{NBS}}^{2} / \underline{\Omega}_{\mathrm{NBS}}$ in watts by equating electrical and mechanical power using a moving-coil force balance [8];

5) determination of $\gamma_{p}^{\prime}$ by the low field method, $\gamma_{\mathrm{p}}^{\prime}$ (low), in terms of the NBS representation of the ampere $A_{\mathrm{NBS}}=V_{\mathrm{NBS}} / \underline{\Omega}_{\mathrm{NBS}}$ using a specially constructed $2.1-\mathrm{m}$ long, single-layer, precision solenoid [9].

Two remarks about these determinations are in order. First, to minimize the contribution of uncertainties associated with laboratory electrical standards to the final uncertainties of the experiments, a great deal of effort is devoted to the problem of standards calibration and monitoring. The goal is to ensure that $\underline{\Omega}_{\mathrm{NBS}}$ and the reference resistors used in the $\gamma_{\mathrm{p}}^{\prime}$ (low) and $W_{\mathrm{NBS}}$ experiments are well known in terms of $R_{\mathrm{H}}$ and that the Zener voltage references used in these two experiments are well known in terms of $E_{J}$. Because of the need for close ties between these artifact standards and the invariant reference resistance and voltage provided by the quantum Hall and Josephson effects, and because the QHE and Josephson effect apparatus are located in a building $1.5 \mathrm{~km}$ from that in which the $\gamma_{p}^{\prime}$ (low) and $W_{\mathrm{NBS}}$ apparatus are located, this aspect of the experiments is not at all trivial. It requires the coordination of a number of different measurement systems and the collaboration and cooperation of many individuals. We are fortunate at NBS to have all of these efforts in one group and believe that the complexity and diversity of these five experiments precludes them from being undertaken at a single institution other than a national standards laboratory.

Second, the values of $R_{\mathrm{H}}$ we shall actually use to evaluate (1) and (2) are based on the same measurements of
$R_{\mathrm{H}}$ in terms of $\underline{\Omega}_{\mathrm{NBS}}$. Consequently, the two resulting values of $\alpha$ are not completely independent and the small correlation between their uncertainties should be considered when comparing them or when combining them to obtain a single "best" value of $\alpha$. Similarly, in principle the value of $E_{\mathrm{J}}$ obtained from the determination of $W_{\mathrm{NBS}}$ is correlated with both values of $\alpha$ through $\underline{\Omega}_{\mathrm{NBS}}$ or $R_{\mathrm{H}}$, and in the case of the value of $\alpha$ obtained from (2), through $V_{\mathrm{NBS}}$ as well. These correlations (and others) are all taken into account in the calculations of Section III as appropriate.

\section{Data, Calculations, and Results}

\section{A. Data}

Since July 1, 1972 [10], the NBS representation of the volt $V_{\mathrm{NBS}}$ has been based on the Josephson effect via the relation

$$
V_{\mathrm{NBS}}=\nu_{\mathrm{J}-\mathrm{NBS}} / E_{\mathrm{J}}
$$

with the following exact adopted value for the Josephson frequency:

$$
\nu_{\mathrm{J}-\mathrm{NBS}}=483593.420 \mathrm{GHz} \text {. }
$$

Beginning February 10, 1987 [5], Josephson arrays have been used to implement this definition of $V_{\mathrm{NBS}}$ with an inherent one standard deviation uncertainty of 0.0089 ppm, where ppm $=$ part-per-million or 1 part in $10^{6}$. (Throughout, all uncertainties are meant to correspond to one standard deviation estimates.)

Based on a realization of the ohm using the NBS calculable capacitor having a mean date of May 17, 1988, it was found that [6]

$$
\underline{\Omega}_{\mathrm{NBS}}=[1-(1.594 \pm 0.022) \mathrm{ppm}] \Omega .
$$

Since August $1983, \underline{\Omega}_{\mathrm{NBS}}$ has been monitored in terms of the quantized Hall resistance $R_{\mathrm{H}}$ via the quantum Hall effect. If we write in analogy with (3a)

$$
\underline{\Omega}_{\mathrm{NBS}}=R_{\mathrm{H}} / r_{\mathrm{K}-\mathrm{NBS}}
$$

where the dimensionless quantity $r_{\mathrm{K} \text {-NBS }}$ is equal to the numerical value of $R_{\mathrm{H}}$ expressed in $\underline{\Omega}_{\mathrm{NBS}}$, then the result of the monitoring may be written as [7]

$$
\begin{aligned}
r_{\mathrm{K}-\mathrm{NBS}}= & 25812.8[1+(1.8420 \pm 0.0116) \mathrm{ppm} \\
& +(0.0529 \pm 0.0040) \\
& \times(t-0.7785) \mathrm{ppm} / \mathrm{yr}]
\end{aligned}
$$

where $t$ is measured in years from January 1, 1987 and the intercept and slope uncertainties are uncorrelated. (The quantity $r_{\mathrm{K}-\mathrm{NBS}}$, where the subscript $\mathrm{K}$ stands for von Klitzing, the discoverer of the QHE, is time dependent because $\underline{\Omega}_{\mathrm{NBS}}$ is based on the mean resistance of five timevarying artifact resistance standards. This is in contrast to $V_{\mathrm{NBS}}$ which is based directly on the Josephson effect rather than on a group of electrochernical cells.)

Measurements of $W_{\mathrm{NBS}}=V_{\mathrm{NBS}}^{2} / \underline{\Omega}_{\mathrm{NBS}}$ using the NBS moving-coil force balance having a mean date of May 15 , 
1988 yielded [8]:

$$
W_{\mathrm{NBS}}=[1-(16.69 \pm 1.33) \mathrm{ppm}] \mathrm{W} .
$$

Measurements of $\gamma_{\mathrm{p}}^{\prime}(\mathrm{low})$ in terms of $T_{\mathrm{NBS}} \propto A_{\mathrm{NBS}}=$ $V_{\mathrm{NBS}} / \underline{\Omega}_{\mathrm{NBS}}$ with a mean date of April 3, 1988 gave the result [9]:

$$
\begin{aligned}
\gamma_{\mathrm{p}}^{\prime}(\text { low })= & 2.67513376(29) \\
& \times 10^{8} \mathrm{~s}^{-1} T_{\mathrm{NBS}}^{-1}(0.11 \mathrm{ppm}) .
\end{aligned}
$$

(Here $T_{\mathrm{NBS}}$, the NBS representation of the tesla, is directly proportional to $A_{\mathrm{NBS}}$ since it is based on a precision solenoid of known dimensions carrying a current known in terms of $A_{\mathrm{NBS}}$.)

\section{B. Calculations}

For numerical convenience, here and throughout the remainder of this paper we consider the inverse fine-structure constant $\alpha^{-1} \simeq 137=1 / \alpha$ rather than $\alpha$. For ease of comparison, we present results in the form $\alpha^{-1}=$ $\sigma_{-1}^{0}[1+(\Delta \pm \epsilon) \mathrm{ppm}]$ which is equivalent to $R_{\mathrm{H}}=R_{0}[1$ $+(\Delta \pm \epsilon) \mathrm{ppm}]$, where $\alpha_{0}^{-1}=2 R_{0} / \mu_{0} c=137.035959$ $5 \cdots$ exactly and $R_{0}=25812.8 \Omega$ exactly. Similarly, we give $E_{\mathrm{J}}=2 e / h$ in the form $E_{\mathrm{J}}=E_{0}\left[1+\left(\Delta^{\prime} \pm \epsilon^{\prime}\right)\right.$ ppm] with $E_{0}=483594 \mathrm{GHz} / \mathrm{V}$ exactly, for ease of comparison with the value suggested by the CCE in 1972 which numerically is $483594 \times 10^{9}$ [11].

With the aid of (5), (1) may be written as

$$
\alpha^{-1}=2 R_{\mathrm{H}} / \mu_{0} c=\left(2 / \mu_{0} c\right) r_{\mathrm{K} \cdot \mathrm{NBS}} \underline{\Omega}_{\mathrm{NBS}}
$$

where it is assumed that the values for $r_{\mathrm{K}-\mathrm{NBS}}$ and $\Omega_{\mathrm{NBS}}$ correspond to the same time $t$. Using the value of $r_{\mathrm{K} \text {-NBS }}$ given by (6) on the mean date of the NBS ohm realization, and the value of $\underline{\Omega}_{\mathrm{NBS}}$ given in (4), we obtain from (9)

$$
\begin{aligned}
\alpha^{-1} & =\alpha_{0}^{-1}[1+(0.280 \pm 0.024) \mathrm{ppm}] \\
& =137.0359979(32) \\
R_{\mathrm{H}} & =25812.80723(61) \Omega .
\end{aligned}
$$

In a similar fashion, with the aid of (3a) and (5), (2) may be written as

$$
\alpha^{-1}=\left\{\left(\mu_{\mathrm{p}}^{\prime} / \mu_{\mathrm{B}}\right) r_{\mathrm{K}-\mathrm{NBS}} \nu_{\mathrm{J}-\mathrm{NBS}} / 2 \mu_{0} R_{\infty} \gamma_{\mathrm{p}}^{\prime}(\text { low })\right\}^{1 / 3}
$$

where as usual \{\} indicates numerical value only. Using the value of $r_{\mathrm{K} \text {-NBS }}$ given by (6) on the mean date of the NBS $\gamma_{\mathrm{p}}^{\prime}$ (low) measurements, the value of $\nu_{\mathrm{J}-\mathrm{NBS}}$ given in (3b), $\gamma_{p}^{\prime}($ low) as given in (8), the 1986 CODATA recommended value for $\mu_{\mathrm{p}}^{\prime} / \mu_{\mathrm{B}}$ (fractional uncertainty = $0.011 \mathrm{ppm}$ ) [3], and $R_{\infty}=10973731.573(4) \mathrm{m}^{-1}$ (fractional uncertainty $=0.0004 \mathrm{ppm}$ ) [12], a more up-to-date and accurate value than that of CODATA, (11) yields

$$
\begin{aligned}
\alpha^{-1} & =\alpha_{0}^{-1}[1+(0.178 \pm 0.037) \mathrm{ppm}] \\
& =137.0359840(51) \\
R_{\mathrm{H}} & =25812.80460(95) \Omega .
\end{aligned}
$$

This result does not agree as well as one would like with that obtained from the realization of the ohm as given in
(10); the two differ by $(0.102 \pm 0.043) \mathrm{ppm}$ or $2.37 \mathrm{com}-$ bined standard deviations. All relevant calculations and corrections are being reviewed in an attempt to understand the significance of this difference.

The values of $\alpha^{-1}$ given in (10) and (12) involve the assumption that $R_{\mathrm{H}}$ is a measure of $h / e^{2}$ and thus of $\alpha^{-1}$ ((12) also requires the assumption $2 e / h=E_{\mathrm{J}}$ ). A value of $\alpha^{-1}$ independent of this assumption (but still requiring the assumption $2 e / h=E_{\mathrm{J}}$ ) can be obtained from our data via the following relation derived by using (1) to eliminate $R_{\mathrm{H}}$ from (2):

$$
\left(\alpha^{-1}\right)^{2}=c\left(\mu_{\mathrm{p}}^{\prime} / \mu_{\mathrm{B}}\right) E_{\mathrm{J}} / 4 R_{\infty} \gamma_{\mathrm{p}}^{\prime}
$$

which may be written as

$\alpha^{-1}=\left\{c\left(\mu_{\mathrm{p}}^{\prime} / \mu_{\mathrm{B}}\right) \nu_{\mathrm{J}-\mathrm{NBS}} / 4 R_{\infty} \underline{\Omega}_{\mathrm{NBS}} \gamma_{\mathrm{p}}^{\prime}(\text { low })\right\}^{1 / 2}$.

For historical reasons, the value of $\alpha^{-1}$ obtained from (13b) is often referred to as the "Josephson junction value of alpha." Using the result

$$
d \underline{\Omega}_{\mathrm{NBS}} / d t=(-0.0529 \pm 0.0040) \mu \Omega / \mathrm{yr}
$$

implied by (6) to correct the value of $\underline{\Omega}_{N B S}$ given in (4) to the mean date of the $\gamma_{p}^{\prime}$ (low) measurements, (3b) for $\nu_{\mathrm{J}-\mathrm{NBS}}$, (8) for $\gamma_{\mathrm{p}}^{\prime}($ low), the 1986 CODATA value for $\mu_{\mathrm{p}}^{\prime} / \mu_{\mathrm{B}}$, and the above value of $R_{\infty}$, we find ${ }^{1}$

$$
\begin{aligned}
\alpha^{-1} & =\alpha_{0}^{-1}[1+(0.127 \pm 0.056) \mathrm{ppm}] \\
& =137.0359770(77) \\
R_{\mathrm{H}} & =25812.80329(145) .
\end{aligned}
$$

Equation (15) also differs from (10) by 2.37 combined standard deviations, as do (12) and (15) with each other. The reason is that the three values are highly interdependent; any two of (9), (11), and (13b) determine the third. (The correlation coefficient of (10) and (12) is 0.040 ; of (10) and (15), -0.170 and of (12) and (15), 0.978.)

To obtain $E_{\mathrm{J}}$ from the measurement of $W_{\mathrm{NBS}}$ in watts, we first use the relation $W_{\mathrm{NBS}}=V_{\mathrm{NBS}}^{2} / \underline{\Omega}_{\mathrm{NBS}}$ and (3a), (5), and (1) to write

$$
\begin{aligned}
E_{\mathrm{J}}^{2} & =\nu_{\mathrm{J}-\mathrm{NBS}}^{2} / \underline{\Omega}_{\mathrm{NBS}} W_{\mathrm{NBS}} \\
& =\nu_{\mathrm{J}-\mathrm{NBS}}^{2} r_{\mathrm{K}-\mathrm{NBS}} / R_{\mathrm{H}} W_{\mathrm{NBS}} \\
& =2 \nu_{\mathrm{J}-\mathrm{NBS}}^{2} r_{\mathrm{K}-\mathrm{NBS}} / \mu_{0} c \alpha^{-1} W_{\mathrm{NBS}} .
\end{aligned}
$$

Using (14) to correct the result for $\underline{\Omega}_{\mathrm{NBS}}$ given in (4) to the mean date of the $W_{\mathrm{NBS}}$ measurements, (3b) for $\nu_{\mathrm{J}-\mathrm{NBS}}$, and (7) for $W_{\mathrm{NBS}}$, we find from (16a)

$$
\begin{aligned}
E_{\mathrm{J}} & =E_{0}[1+(7.94 \pm 0.67) \mathrm{ppm}] \\
& =483597.84(32) \mathrm{GHz} / \mathrm{V} .
\end{aligned}
$$

'Taking $d \underline{\Omega}_{\text {NBS }} / d t$ from quantized Hall resistance measurements does not negate the independence of this value of $\alpha^{-1}$ from the assumption $R_{\mathrm{H}}$ $=h / e^{2}$. The only assumption required is that $R_{\mathrm{H}}$ is time-independent. 
Alternatively, we may use (16b) with $r_{\mathrm{K}-\mathrm{NBS}}$ from (6) and $R_{\mathrm{H}}$ from $(12 \mathrm{c})$. The result is

$$
\begin{aligned}
E_{\mathrm{J}} & =E_{0}[1+(7.99 \pm 0.67) \mathrm{ppm}] \\
& =483597.87(32) \mathrm{GHz} / \mathrm{V} .
\end{aligned}
$$

If we repeat this calculation using $R_{\mathrm{H}}$ from (15c), we obtain

$$
\begin{aligned}
E_{\mathrm{J}} & =E_{0}[1+(8.02 \pm 0.67) \mathrm{ppm}] \\
& =483597.88(32) \mathrm{GHz} / \mathrm{V} .
\end{aligned}
$$

The differences among these three values of $E_{\mathrm{J}}$ see ((17)(19)) are small compared to the uncertainties of the three values, which are completely dominated by the $1.33-\mathrm{ppm}$ uncertainty of $W_{\mathrm{NBS}}$. Hence, the differences among the values of $R_{\mathrm{H}}$ discussed above are not critical to deriving a value of $E_{\mathrm{J}}$ from $W_{\mathrm{NBS}}$.

\section{Final Results}

To obtain a single best value of $\alpha^{-1}$ (or equivalently, $R_{\mathrm{H}}$ ) and of $E_{\mathrm{J}}$ from the NBS data, and subsequently values of $h$ and $e$ from these best values, we use the method of least squares as employed in fundamental constants adjustments [3]: Taking $\alpha^{-1}$ and $E_{\mathrm{J}}$ as the adjustable constants or unknowns, the observational equations for the three measured quantities $\underline{\Omega}_{\mathrm{NBS}}, \gamma_{\mathrm{p}}^{\prime}$ (low), and $W_{\mathrm{NBS}}$ are

$$
\begin{aligned}
& \alpha^{-1}=\left(2 / \mu_{0} c\right) r_{\mathrm{K}-\mathrm{NBS}} \underline{\Omega}_{\mathrm{NBS}} \\
& \alpha^{-3}=\left\{\left(\mu_{\mathrm{p}}^{\prime} / \mu_{\mathrm{B}}\right) r_{\mathrm{K}-\mathrm{NBS}} \nu_{\mathrm{J}-\mathrm{NBS}} / 2 \mu_{0} R_{\infty} \gamma_{\mathrm{p}}^{\prime}(\text { low })\right\}
\end{aligned}
$$

$$
\alpha^{-1} E_{\mathrm{J}}^{2}=2 \nu_{\mathrm{J}-\mathrm{NBS}}^{2} r_{\mathrm{K}-\mathrm{NBS}} / \mu_{0} c W_{\mathrm{NBS}}
$$

which follow from (9), (11), and (16c). Then since $R_{\mathrm{H}}=$ $\mu_{0} c \alpha^{-1} / 2=h / e^{2}$ and $E_{\mathrm{J}}=2 e / h^{2}$

$$
\begin{aligned}
& h=4 / R_{\mathrm{H}} E_{\mathrm{J}}^{2} \\
& e=2 / R_{\mathrm{H}} E_{\mathrm{J}} .
\end{aligned}
$$

It is apparent from (20) and (6) that $r_{\mathrm{K}-\mathrm{NBS}}(t=0)$ and $d r_{\mathrm{K}-\mathrm{NBS}} / d t$ (i.e., the intercept and slope of (6)) could be taken as adjustable constants in addition to $\alpha^{-1}$ and $E_{\mathrm{J}}$. We choose not to for the following reason: because of their comparative simplicity and the extended period over which they have been carried out (i.e., about five years), we believe that at present, our quantized Hall resistance measurements are inherently more reliable than our other measurements (i.e., $\underline{\Omega}_{\mathrm{NBS}}$ and $\gamma_{\mathrm{p}}^{\prime}(\mathrm{low})$ ), more so perhaps than is indicated by their a priori assigned uncertainties. Consequently, we do not believe $r_{\mathrm{K}-\mathrm{NBS}}(t=0)$ or $d r_{\mathrm{K}-\mathrm{NBS}} / d t$ should be subject to adjustment. Moreover, because of the structure of (20a)-(20c), taking these two quantities as adjustable constants yields identical values for $\alpha^{-1}$ and $E_{\mathrm{J}}$. In addition, the adjusted value of $r_{\mathrm{K}-\mathrm{NBS}}(t$

\footnotetext{
${ }^{2}$ In principle, to calculate properly the uncertainty of $h$ and $e$, the covariance of $\alpha^{-1}$ and $E_{\mathrm{J}}$, which is one of the outputs of the adjustment, must be taken into account.
}

$=0$ ) would be only 0.0034 ppm smaller than the input value from (6) (0.3 standard deviations of the latter); and the adjusted value of $d r_{\mathrm{K}-\mathrm{NBS}} / d t$ would be only 0.0004 $\mathrm{ppm} / \mathrm{yr}$ smaller than the input value from (6) (0.1 standard deviations of the latter).

To solve (20a)-(20c), we use: 1) (6) to calculate $r_{\mathrm{K}-\mathrm{NBS}}$ at the mean dates of the $\underline{\Omega}_{\mathrm{NBS}}, \gamma_{\mathrm{p}}^{\prime}\left(\right.$ low), and $W_{\mathrm{NBS}}$ measurements as before, 2) the values given above for these quantities as well as for $\nu_{\mathrm{J}-\mathrm{NBS}}, \mu_{\mathrm{p}}^{\prime} / \mu_{\mathrm{B}}$, and $R_{\infty}$, and 3 ) the correlated error least squares approach [13]. This latter method is employed to take into account the approximate $0.012-\mathrm{ppm}$ uncertainty in $r_{\mathrm{K}-\mathrm{NBS}}$ common to all three equations; some comparatively small systematic uncertainties associated with resistance measurements common to the $R_{\mathrm{H}}, \underline{\Omega}_{\mathrm{NBS}}, \gamma_{\mathrm{p}}^{\prime}\left(\right.$ low), and $W_{\mathrm{NBS}}$ experiments; and for completeness the 0.0089 -ppm uncertainty in implementing the definition of $V_{\mathrm{NBS}}$ common to (20b) and (20c). (All of these were also taken into account in the calculations of the previous section.)

The difference between the values of $\alpha^{-1}$ from $\underline{\Omega}_{\mathrm{NBS}}$ and $\gamma_{p}^{\prime}($ low) indicated above (see (10) and (12)) is apparent in the least-squares treatment; the statistic "chi square" $\left(\chi^{2}\right)$ for the adjustment is 5.60 compared with its expected value of $f=1$ ( $f$ is the number of degrees of freedom ); the Birge ratio is $R_{\mathrm{B}}=\left[\chi^{2} / f\right]^{1 / 2}=2.37 \mathrm{com}$ pared with its expected value of 1 . The probability that this value of $\chi^{2}$ has occured by chance is about 1.8 percent. The resulting adjusted value of $\alpha^{-1}$ is $\alpha_{0}^{-1}[1+$ $(0.252 \pm 0.020) \mathrm{ppm}]$.

The above value of $\chi^{2}$ arises entirely from the difference between the two values of $\alpha^{-1} ; W_{\mathrm{NBS}}$ does not contribute to $\chi^{2}$ or to the determination of $\alpha^{-1}$ since $\alpha^{-1} E_{\mathrm{J}}^{2}$ $\propto 1 / W_{\text {NBS }}$ (see (20c)) and (20a) and (20b) do not involve $E_{\mathrm{J}}$. Thus a one variable (i.e., $\alpha^{-1}$ ) least squares adjustment involving just $\underline{\Omega}_{\mathrm{NBS}}$ and $\gamma_{\mathrm{p}}^{\prime}($ low) ((20a) and (20b)) yields the same adjusted value for $\alpha^{-1}$ and also has $\chi^{2}=$ 5.60 for $f=1$ with $R_{B}=2.37$. If $R_{\mathrm{B}}=2.37$ is used as a multiplicative scale factor for the uncertainties of the one variable adjustment data so that $\chi^{2}$ has its expected value of $1,{ }^{3}$ and if the two variable (i.e., $\alpha^{-1}$ and $E_{\mathrm{J}}$ ) adjustment is then repeated with these expanded uncertainties for (20a) and (20b) with the result that $\chi^{2}$ for this adjustment also has its expected value of 1 , we find

$$
\begin{aligned}
\alpha^{-1} & =\alpha_{0}^{-1}[1+(0.252 \pm 0.048) \mathrm{ppm}] \\
& =137.0359940(65) \\
R_{\mathrm{H}} & =25812.80650(123) \Omega \\
E_{\mathrm{J}} & =E_{0}[1+(7.96 \pm 0.67) \mathrm{ppm}] \\
& =483597.85(32) \mathrm{GHz} / \mathrm{V} \\
h & =6.6260704(88) \times 10^{-34} \mathrm{~J} \cdot \mathrm{s}(1.33 \mathrm{ppm})
\end{aligned}
$$

${ }^{3}$ The adjusted value of $\alpha^{-1}$ is unchanged but its uncertainty is increased to $0.048 \mathrm{ppm}=2.37 \times 0.020 \mathrm{ppm}$. 


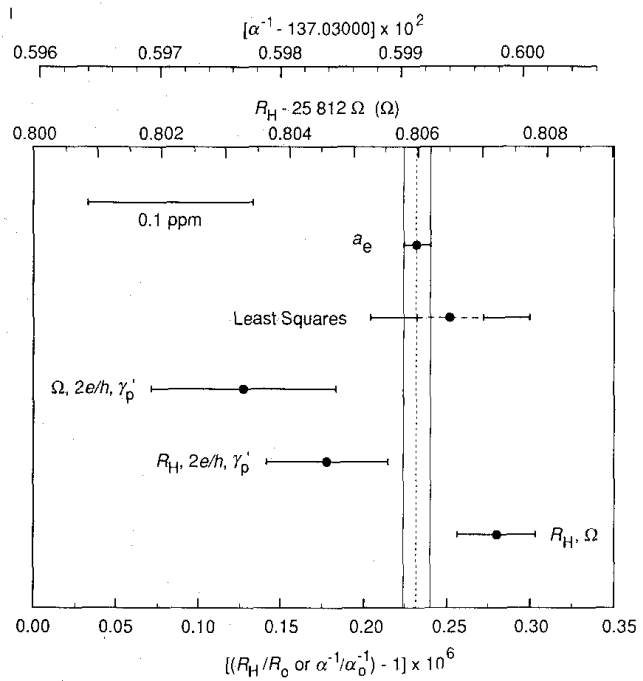

Fig. 1. Comparison of values of $R_{\mathrm{H}}$ and $\alpha^{-1}$ discussed in the text. ( $R_{0}=$ $25812.8 \Omega, \alpha_{0}^{-1}=2 R_{0} / \mu_{0} c=137.0359595 \cdots$.) Starting from the bottom of the figure, the equation number of the value as given in the text is: (10), (12), (15), (22), (26). The least squares value, (22), is shown with and without its uncertainty increased by the scale factor $R_{\mathrm{B}}$ $=2.37$; see text. (As a comparison aid, the most accurate value $(26)$ and its uncertainty are indicated by dashed and full lines, respectively, as well as by the usual point and error bars.)

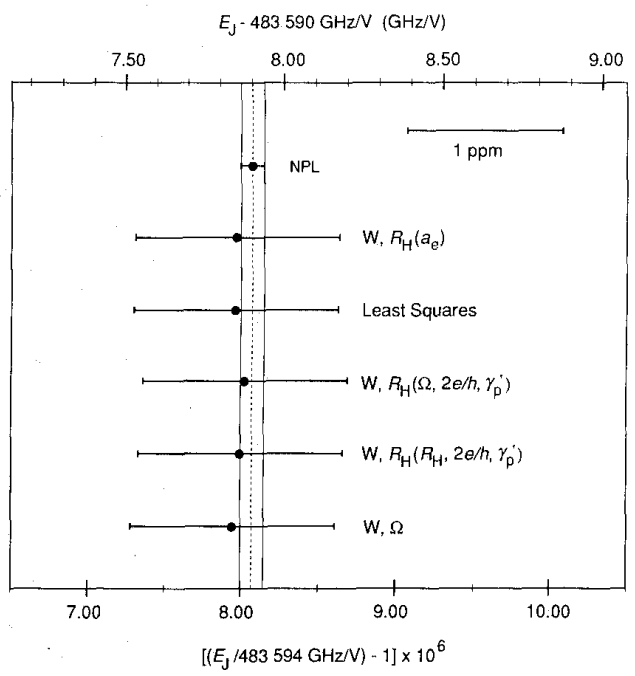

Fig. 2. Comparison of values of $E_{\mathrm{I}}$ discussed in the text. Starting from the bottom of the figure, the equation number of the value as given in the text is: (17)-(19), (23), (27), (28). (As a comparison aid, the most accurate value (28) and its uncertainty are indicated by dashed and full lines, respectively, as well as by the usual point and error bars.)

$$
e=1.60217670(107) \times 10^{-19} \mathrm{C}(0.67 \mathrm{ppm}) .
$$

In Figs. 1 and 2 we graphically compare the principal values of $\alpha^{-1}$ and $E_{\mathrm{J}}$ obtained throughout this paper from our data. Also included in Fig. 1 is the highly accurate but still preliminary QED value of $\alpha^{-1}$ obtained by Kinoshita from the electron magnetic moment anomaly $a_{\mathrm{e}}$
[14]:

$$
\begin{aligned}
\alpha^{-1}\left(a_{\mathrm{e}}\right) & =\alpha_{0}^{-1}[1+(0.2321 \pm 0.0080) \mathrm{ppm}] \\
& =137.0359914(11) \\
R_{\mathrm{H}} & =25812.80599(21)
\end{aligned}
$$

If this value of $R_{\mathrm{H}}$ is used to calculate a value of $E_{\mathrm{J}}$ from (16b), the result is

$$
\begin{aligned}
E_{\mathrm{J}} & =E_{0}[1+(7.97 \pm 0.67) \mathrm{ppm}] \\
& =483597.85(32) \mathrm{GHz} / \mathrm{V} .
\end{aligned}
$$

This value is also included in Fig. 2 as is the most precise result reported to date, that obtained by Kibble et al. [15] at the National Physical Laboratory (NPL), U.K., from their version of the moving-coil watt realization experiment:

$$
\begin{aligned}
E_{\mathrm{J}} & =E_{0}[1+(8.070 \pm 0.077) \mathrm{ppm}] \\
& =483597.903(37) \mathrm{GHz} / \mathrm{V} .
\end{aligned}
$$

It should be borne in mind while examining Figs. 1 and 2 , especially Fig. 2, that the NBS values are highly interdependent. Indeed, all the values of $E_{\mathrm{J}}$ except that of NPL are essentially the same value since the uncertainty of $W_{\mathrm{NBS}}$ is so overwhelmingly dominant.

\section{Conclusion}

We have presented a detailed analysis of the currently available NBS results from ongoing experiments to realize the ohm, to realize the watt, to determine the proton gyromagnetic ratio by the low field method, to determine the time dependence of the NBS representation of the ohm using the quantum Hall effect, and to maintain the NBS representation of the volt using Josephson 1-V arrays. Either of our values for $\alpha^{-1}$ and thus $R_{\mathrm{H}}$ in SI units as derived from (1) and (2) (see (10) and (12)) is more accurate than any QED-independent value presently available. This is even true of our least-squares adjusted value with expanded uncertainty, (22). All three values agree with the highly accurate QED value of $\alpha^{-1}$ from the electron magnetic moment anomaly, (26), since the difference from $\alpha^{-1}\left(a_{e}\right)$ for each is less than two combined standard deviations. However, the two values derived from (1) and (2) differ from each other by somewhat more than two combined standard deviations. Every effort will be made in the near future to understand if this difference is significant.

Our values for $E_{1}, h$, and $e$ have rather larger uncertainties than their 1986 CODATA recommended counterparts, although they are quite consistent with them. However, it is expected that when the room-temperature magnetic field-generating solenoid of the NBS watt realization experiment is replaced by a specially constructed superconducting solenoid, the uncertainties of the NBS values will be reduced by between a factor of 15 and 50 . This would make them the most accurate values available. 


\section{REFERENCES}

[1] T. Kinoshita, "The anomalous magnetic moment of the electron and the quantum electrodynamics determination of the fine-structure constant," IEEE Trans. Instrum. Meas., vol. IM-36, pp. 201-204, June 1987.

[2] B. N. Taylor, "Impact of quantized Hall resistance on SI electrical units and fundamental constants," Metrologia, vol. 21, pp. 37-39, 1985.

[3] E. R. Cohen and B. N. Taylor, "The 1986 adjustment of the fundamental physical constants," Rev. Mod. Phys., vol. 59, no. 4, pp. $1121-1148$, Oct. 1987.

[4] P. Giacomo, "News from the BIPM," Metrologia, vol. 24, pp. 45$51,1987$.

[5] R. L. Steiner and B. F. Field, "Josephson array voltage calibration system: Operational use and verification," IEEE Trans. Instrum. Meas., pp. 296-301, this issue.

[6] J. Q. Shields, R. F. Dziuba, and H. P. Layer, "New realization of the ohm and farad using the NBS calculable capacitor," IEEE Trans. Instrum. Meas., pp. 249-251, this issue.

[7] M. E. Cage, R. F. Dziuba, C. T. Van Degrift, and D. Y. Yu, "Determination of the time dependence of $\Omega_{\mathrm{NBS}}$ using the quantized Hall resistance,"' IEEE Trans. Instrum. Meas., pp. 263-269, this issue.
[8] P. T. Olsen, R. E. Elmquist, W. D. Phillips, E. R. Williams, G. R. Jones, Jr., and V. E. Bower, "A measurement of the NBS electrical watt in SI units," IEEE Trans. Instrum. Meas., pp. 238-244, this issue.

[9] E. R. Williams, G. R. Jones, Jr., Ye Sheng, Liu Ruimin, H. Sasaki, P. T. Olsen, W. D. Phillips, and H. P. Layer, "A low field determination of the proton gyromagnetic ratio in $\mathrm{H}_{2} \mathrm{O}$," IEEE Trans. Instrum. Meas., pp. 233-237, this issue.

[10] B. F. Field, T. F. Finnegan, and J. Toots, "Volt maintenance at NBS via $2 e / h$ : A new definition of the NBS volt," Metrologia, vol. 9 , pp. $155-166 ; 1973$.

[11] J. Terrien, "News from the Bureau International des Poids et Mesures," Metrologia, vol. 9, pp. 40-43, 1973.

[12] P. Zhao, W. L. Lichten, H. Layer, and J. Bergquist, "New value for the Rydberg constant from the hydrogen Balmer- $\beta$ transition," Phys. Rev. Lett., vol. 58, no. 13, pp. 1293-1295, Mar. 1987; Erratum, no. 23 , p. 2506, June 1987.

[13] M. G. Natrella, Experimental Statistics, National Bureau Standard, Handbook 91 USGPO, Washington, DC, 1963, pp. 6-22-6-26.

[14] T. Kinoshita, "Accuracy of the fine-structure constant," IEEE Trans. Instrum. Meas., pp. 172-175, this issue.

[15] B. P. Kibble, I. A. Robinson, and J. H. Belliss, "A realization of the SI watt by the NPL moving-coil balance,'” NPL Rep. DES 88 ; National Physical Lab., Teddington, U.K., May 1988, 54 p. 